\title{
La influencia de los medios en la formación de la opinión pública: los procesos jurídicos y los juicios paralelos ${ }^{1}$
}

\section{Fermín Bouza}

Catedrático de Opinión Pública.

Universidad Complutense

\section{Resumen:}

La presión mediática sobre el sistema judicial alcanza porcentajes de noticias en portada muy significativos. La opinión pública sobre la justicia en España no es buena, y ello es posible atribuirlo, en parte, a esa presión mediática. A pesar de tal presión, la Agenda Pública no recoge una preocupación significativa por la Justicia (tampoco la Agenda Público-Personal o Agenda Personal). No hay una transferencia importante de la Agenda Mediática a la Agenda Pública, siguiendo la terminología de la teoría de la Agenda Setting, y no hay, por tanto, un área de impacto (conjunto-intersección de las agendas pública y personal en los tres primeros rangos de ambas) que incluya en ningún caso a la Justicia y sus problemas, que no es nunca un problema o tema significativo en las agendas pública y personal

Palabras clave:

Opinión pública - medios de comunicación - justicia - Agenda Setting - área de impacto - agenda pública - agenda personal.

\section{Abstract:}

Media pressure on the judicial system is taking up very significant percentages of front page news. Public opinion about justice in Spain is not good and this can be attributed partly to this media pressure. Despite

${ }^{1}$ Esta investigación y este artículo se prepararon, en parte, para el curso sobre Justicia y Medios de Comunicación que se desarrolló en el Consejo General del Poder Judicial del 31 de Mayo al 2 de Junio de 2006. 
this pressure, the Public Agenda does not include a significant preoccupation with Justice (nor do the Personal-Public Agenda or the Personal Agenda). There is no important transfer from the Media Agenda to the Public Agenda, following the terminology of the theory of Agenda setting, and there is, therefore, no impact area (intersection-set of the public and personal agendas in the three highest rankings of both) that includes Justice and its problems in any way; this is never a significant problem or issue on the public and personal agendas.

Key words:

Public Opinión, Communication Media, Justice, Agenda Setting, Impact Area, Publica Agenda, Personal Agenda.

\section{Objetividad y procesos de decisión judicial}

La actividad judicial, fundada en la independencia -que es base fundamental del sistema jurídico-, no es una variable independiente y autónoma que los sociólogos podamos analizar al margen de los procesos reales que nos la muestran como variable altamente compleja en el conjunto de variables (entre ellas, la opinión pública) que concurren en el proceso de decisión judicial. Tampoco la percepción popular le concede ese estatus de independencia y autonomía de forma mayoritaria (ver tabla 7 y tabla 8), y con frecuencia tiende a exagerar hasta lo inaceptable los rasgos más percibidos como dependientes de la conducta judicial. Pero todo ello forma parte de la complejidad de la propia función judicial en las sociedades de opinión pública, en las sociedades democráticas entre las que tenemos la fortuna de contarnos.

En el campo de la ciencia, la objetividad (atributo central de la función judicial) no es tampoco una variable al margen del mundo: el método científico construye un espacio intersubjetivo de trabajo, pero no es la objetividad misma, que en su versión estricta es, por definición, un ente teórico: el método científico garantiza esa intersubjetividad suficiente (el método, a modo de imperativo categórico kantiano, garantiza ese Sujeto Universal de la ciencia que hace posible la comunicación y el saber mismo) y nos obliga a trabajar entre ciertos límites regulados que nos acercan a algo que, de modo retórico (y sólo retórico) llamamos objetividad. También las garantías jurídicas tienden a crear esa "objetividad" metodológica, que es la única objetividad posible. Por tanto, el trabajo científico, como el jurídico, no busca un juicio o una experiencia sin sujeto (un sujeto que se postula cargado de buenas intenciones, por qué no): busca la objetividad metodológica que garantice el control regulado del proceso científico o jurídico: es, y sobre todo es, una cuestión de método. Y mejorar el método es el objetivo de toda evaluación de las conductas científicas o de la evaluación de las dependencias de los sujetos jurídicos en los procesos de decisión judicial, como en este caso que me ocupa, el de los juicios paralelos y la opinión pública. Es decir: se trata de la mejora del método sobre la base del conocimiento de los pro- 
cesos reales de formación de la decisión jurídica, no de la desaparición del sujeto científico o jurídico (la desaparición de lo subjetivo), que es simplemente absurda.

Las "formas no reguladas de control judicial" (voy a definir así al tipo de variables extrañas al propio proceso judicial) tienen en la movilización y/o manipulación de la Opinión Pública su principal instrumento. Como sociólogo, y sobre todo como sociólogo especializado en el análisis de la Opinión Pública, voy a tratar de situar esta cuestión en la tradición analítica sobre los "efectos de los medios de comunicación”, y desde ahí interpretar algunos datos empíricos relativos al tratamiento en España de la cuestión judicial.

Quizá no es necesario aclarar que lo que llamo "movilización y/o manipulación de la Opinión Pública" es algo perfectamente legítimo en democracia y en los márgenes que concede la ley, y no es necesariamente un acto reprobable o ilegítimo. Conviene recordarlo porque la palabra "manipulación", que en el campo de las Ciencias Sociales se usa en general de forma neutra, técnica, en la retórica convencional alude a algo negativo o muy negativo. En este sentido, la presión sobre el poder judicial se ejerce desde frames (es la palabra inglesa habitual, aquí traducida indistintamente como "marcos" o “encuadres") políticos de la realidad, y responde a una dinámica de esa clase, política, en la que la permisividad enunciativa y narrativa (libertad de expresión política) desborda con frecuencia los presupuestos en que se funda la libertad de expresión en sentido jurídico estricto, mucho más allá de lo que se le permitiría al sujeto individual expresando opiniones individuales sobre instituciones o personas. Pero la política permite esos excesos en la tradición democrática, y es poco frecuente el recurso a la acción judicial contra alguna de esas presiones y opiniones, que también con frecuencia insinúan de forma muy directa graves delitos contra personas e instituciones.

Las "actividades judiciales" están muy presentes en las portadas de los diarios (más adelante explicaré la metodología usada), en una media del 25,23\% (en estas cifras, los decimales son una consecuencia del cálculo, no algo que haya que leer de forma estricta) del total de noticias.

Tabla 1

Noticias Judiciales en portada

(\% interior al propio diario: 10-03-2006 a 24-03-2006, ambos incluidos)

\begin{tabular}{|l|l|}
\hline El Mundo & 29,20 \\
\hline El País & 29,01 \\
\hline ABC & 28,42 \\
\hline El Periódico & 11,83 \\
\hline TODOS & 25,23 \\
\hline
\end{tabular}




\section{Gráfico 1 \\ \% en cada diario de noticias judiciales en portada 10/03/06 a 24/03/06, ambos incluidos}

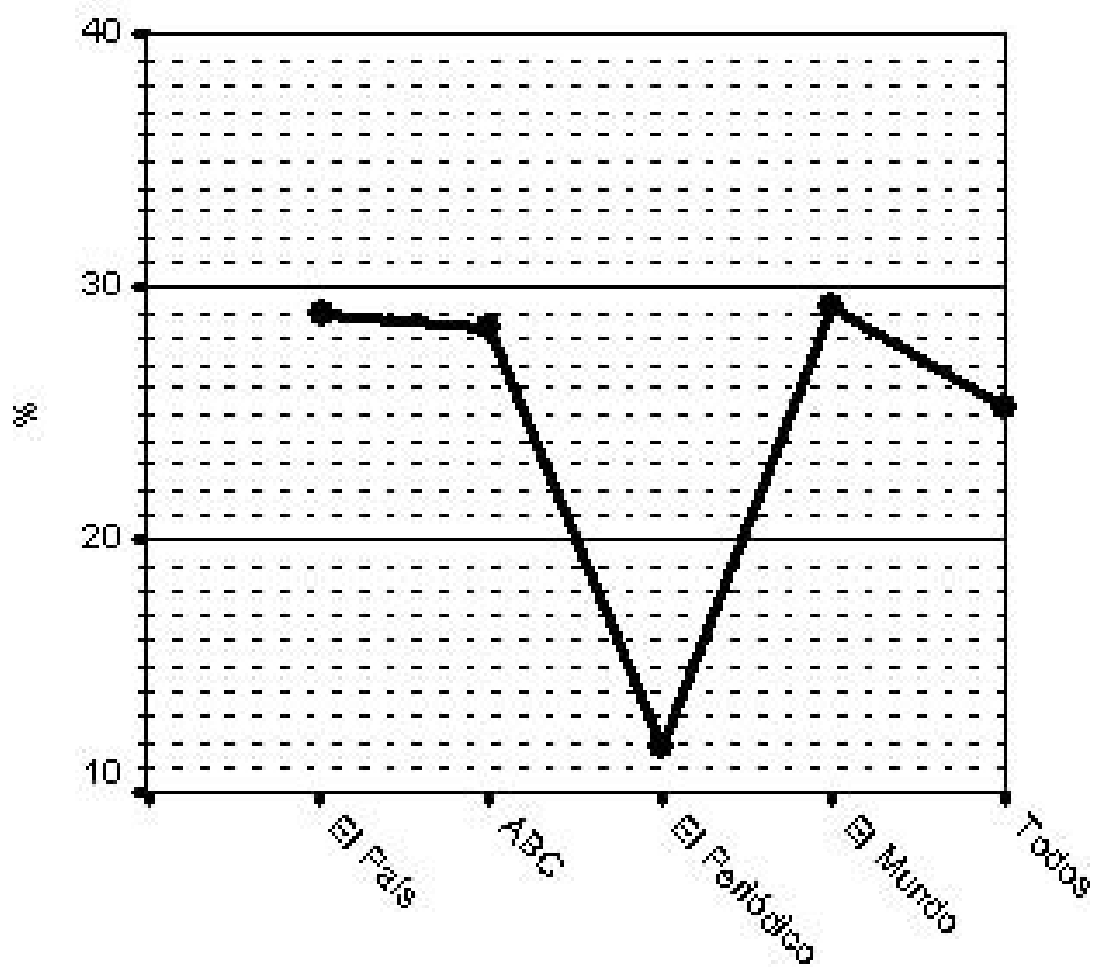

\section{Diario}

A su vez, de esas noticias de actividades judiciales, cerca del $40 \%(37,61 \%$, exactamente, y en algún caso, como el de El Mundo, con un porcentaje del 42,42\%) lo son sobre las actividades judiciales en sí mismas (aquí se llaman "vínculos judiciales"): juicios y presiones sobre los jueces y sobre el mundo judicial: 


\section{Tabla 2}

\% de vínculos judiciales sobre el total de noticias judiciales de portada en cada diario (vínculos judiciales: noticias judiciales que se centran en el trabajo judicial) y desviación porcentual de la media (10/03/2006 a 24/03/2006)

\begin{tabular}{|l|l|}
\hline El Mundo & $42,42(+4,19)$ \\
\hline ABC & $40,74(+3,13)$ \\
\hline El Periódico & $36,36(-1,25)$ \\
\hline El País & $31,58(-6,03)$ \\
\hline TODOS & 37,61 (Media) \\
\hline
\end{tabular}

Gráfico 2

\% de vínculos judiciales en las noticias judiciales de portada 10/03/06 a 24/03/06

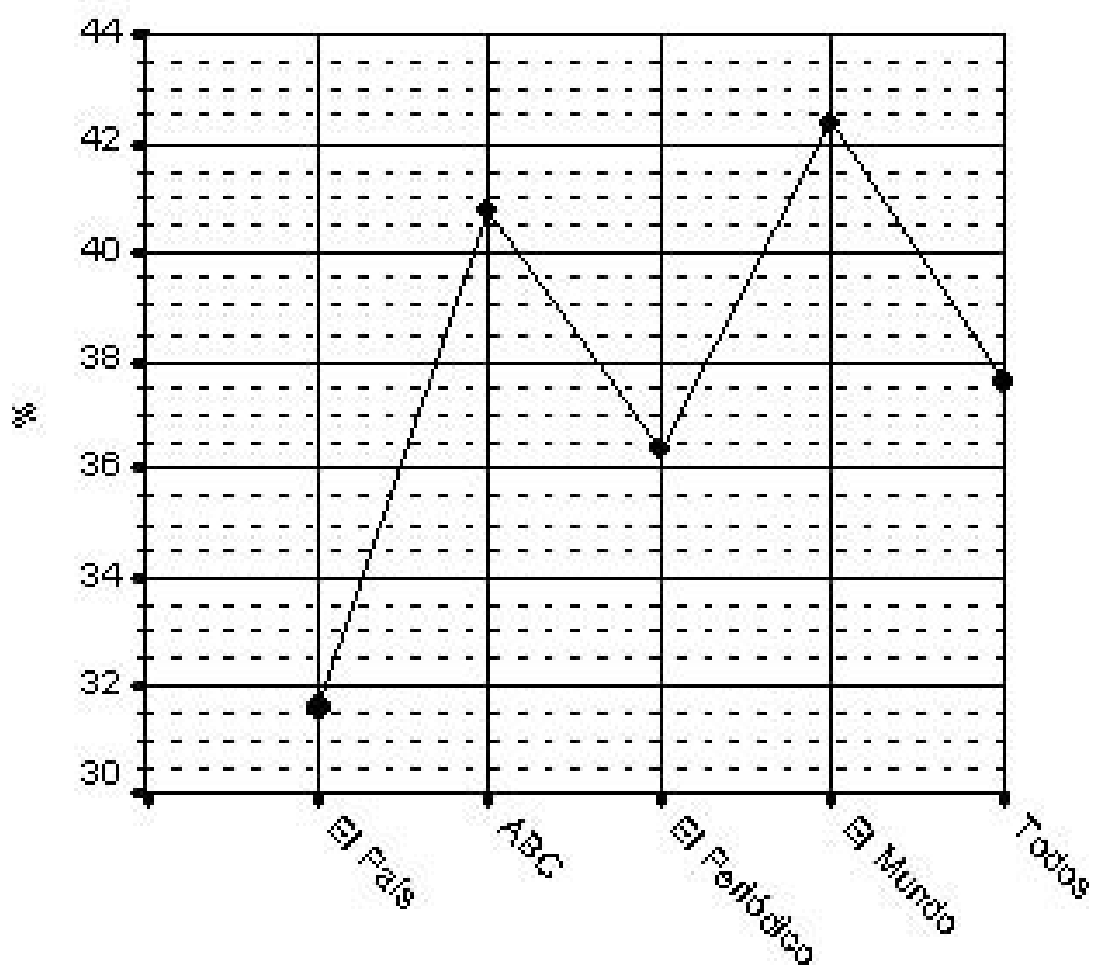

Diario 
Así las cosas, cabe decir que el mundo judicial soporta una de las mayores presiones mediáticas en nuestro país. ¿Es posible trabajar a gusto bajo tal fuego mediático? No digo yo que eso no tenga un aspecto satisfactorio, pues sin duda el que hablen de uno (aunque sea bien, como dice el dicho popular) puede resultar gratificante, pero también cansino y paralizante, cuando no directamente mediatizador, en el peor sentido. Algunos casos recientes en diversos países de Europa remiten siempre a la misma conclusión: la aceptación como un bien superior de la libertad periodística para informar de las actividades judiciales, opinar y juzgar sobre ellas ${ }^{2}$.

Para obtener los datos en los que me baso, seguí estas normas de trabajo:

\section{Metodología}

Se hizo un seguimiento de la prensa escrita del 10/03/06 al 24/03/06 (ambos inclusive) a través de cuatro diarios, dos más próximos ideológicamente al gobierno actual, y dos más alejados (El País, El Periódico, El Mundo y ABC). Se computaron solo noticias de portada que no hicieran referencia a suplementos, aunque sí a partes interiores del periódico, siempre que esto apareciera claro y no cupiera confusión con suplementos (no siempre es así). Prescindo de promociones y similares. Son noticias en sentido estricto. Si hubiera alguna vacilación o irregularidad debido a la complejidad, a veces, de la elección, estas deben ser menores e irrelevantes para las cifras del cómputo. Se computan las actividades policiales con orden judicial (se excluyen las puramente policiales: la represión contra el botellón, por ejemplo, y no se tiene en cuenta el tema OPA-Endesa-Gas Natural-Eon y similares (Tribunal de Cuentas) si no hay alusiones judiciales. De las actividades judiciales se distinguen las que tienen que ver con el terrorismo, la política, la economía, y "las criminales y otras", así como las judiciales en sí mismas (críticas a jueces, o presiones sobre el sumario, por ejemplo) Se computa, en el interior de las actividades judicialesjudiciales, las que tienen que ver con el terrorismo (son el mayor número en el conjunto de las actividades judiciales públicas, y se trata de saber su porcentaje, que se ve afectado al separar las judicialesjudiciales: por eso se hace el cómputo de judiciales-judiciales-terroristas), aunque en algunos casos esas tablas y números no figuren en este artículo.

\footnotetext{
${ }^{2}$ Azurmendi, Ana (2000): "Tribunal Europeo de derechos humanos (1959-1999): cuarenta años de jurisprudencia sobre el derecho a la información”, Comunicación y Sociedad, vol. XIII, n. ${ }^{\circ}$ 1: http://www.unav.es/cys/xiiil/proteger/azur.htm\#ftn50: (en apartado 7) "En esta ocasión el Tribunal Europeo declaró que, sin traspasar los límites fijados por una buena administración de justicia", "la prensa es competente para informar sobre cuestiones que afecten a los tribunales y al interés público, más aún cuando esta función de comunicación está en íntima relación con el derecho del ciudadano a recibirla y a estar informado" (Sunday Times 54, p. 33). Queda claro que convertir en espectáculo una crónica de tribunales distorsiona la función de comunicar sobre asuntos de interés general y puede llegar a distorsionar la misma administración de justicia. Y es esto, la espectacularización, lo que el Tribunal considera inadmisible en el ejercicio del periodismo. Pero informar acerca de materias que son de interés para una mayoría de ciudadanos, aun cuando éstas sean objeto de un proceso judicial, es, y así lo expresa el mismo órgano jurisdiccional europeo, una competencia de los medios de comunicación en su actividad periodística. Se exige por lo tanto al profesional de la comunicación tener la medida justa del cómo informar sobre estos acontecimientos, y esto en función del derecho del ciudadano a la información.
} 
En caso de ambigüedad (política/economía, un ejemplo que es frecuente) se resuelve a favor de la materia inmediata (economía, generalmente, en el caso citado). Cuando van dos o más noticias en una, se considera la noticia global si no hubiera otros problemas significativos de cómputo (como declaraciones divergentes sobre la misma cuestión, entonces se computarían tantas noticias como declaraciones, o que las subnoticias sean clara y significativamente distintas al titular). En el tema terrorismo se han incluido actividades directa e indirectamente relacionadas con el terrorismo en el contexto de la noticia. Cuando los editoriales comienzan en la portada se computan como noticia. En general, no hay problemas de cómputo, pero con alguna frecuencia hay que tomar decisiones más o menos complejas para excluir o incluir una noticia, o para hacerlo en el marco de según qué rótulo. Ninguna de estas elecciones, en el caso de que fueran discutibles o erróneas, variarían de forma significativa el resultado final de la contabilidad realizada.

Estas muestras son solo indicativas, y no responden al mismo fundamento estadístico de una encuesta de opinión, ni garantizan nada más allá de las cifras puntuales que recogen: no son la parte de una totalidad estática equitemporal, sino un momento de una totalidad que nunca llega a existir como tal. Son un procedimiento experimental o empírico, más que propiamente estadístico. En este caso, el tramo analizado lo ha sido por la simple razón de que es el momento en el que yo mismo comencé a preparar esta conferencia para cumplimentar la invitación a este ciclo en la sede del CGPJ. Una razón de azar como cualquier otra, pero no una garantía de que siempre sean así las cifras expuestas, aunque es muy probable que lo sean en similar periodo activo del año judicial, dejando a un lado periodos vacacionales de baja actividad política (cuestión central en las noticias de "actividades judiciales") y/o judicial.

\section{Des-mediatizando la justicia}

Un sugerente trabajo sobre Ruanda y el caso de los medios de comunicación que indujeron la comisión de crímenes, que citaré alguna vez más de ahora en adelante, lleva por título Desmediatizando el caso de los Medios, o De-Mediatizing the Media Case (Media: medios de comunicación). De ahí tomo la palabra para encabezar este apartado. Observa la autora que cuando se mediatiza un caso judicial como el que ella estudia, que tiene que ver directamente con el papel de los medios y atañe directamente a la libertad de expresión, los organismos internacionales deben proceder con el nivel más alto de exactitud en el desarrollo del razonamiento legal y en la determinación de los estándares requeridos de evidencia ${ }^{3}$.

\footnotetext{
${ }^{3}$ Della Morte, Gabriele (2005): p. 1128:

"...because of the freedom of speech issues underlying the present case, the Media case has been immediately mass-mediatized. Such circumstances, though, should prompt, rather than discourage, a critical appraisal of the Judgment. In such sensitive areas, international bodies should proceed with the highest level of accuracy in developing legal reasoning and determining required standards of evidence." ${ }^{3}$ (...debido a los cuestiones sobre la libertad de expresión que subyacen al presente caso, este caso
} 
Eso podría ser una buena noticia: puesto que los medios están ahí y representan la más obvia y pública concreción de la libertad de expresión, y la justicia en general debe verse afectada para ser, si cabe, más rigurosa, más exigente, más convincente. ¿Es así? Sería arriesgado hacer una afirmación de cualquier signo, pero lo que los ciudadanos parecen percibir no es tan optimista en estas respuestas al barómetro del CIS de Diciembre de 2005 (se seleccionan las cuestiones más significativas) ${ }^{4}$ :

La Administración de Justicia es la peor valorada de las instituciones que se proponen (Voy a leerle ahora el nombre de diferentes instituciones. Qué grado de confianza le merece a usted cada una de ellas: mucha confianza, bastante, poca o ninguna confianza? Se trata del Consejo General del Poder Judicial, las Fuerzas de Seguridad del Estado -Policía y Guardia Civil-, el Defensor del Pueblo, el Tribunal Constitucional y la Administración de Justicia), y este dato global podemos ampliarlo y profundizarlo cruzándolo por la variable "autoubicación política" (y hay que lamentar que en este barómetro del CIS no aparezca la variable "ingresos en el hogar" para poder cruzarla, lo que hubiera sido de gran interés).

En la tabla 3 tenemos el cruce de "autoubicación" con la respuesta al ítem de la justicia, y podemos observar cómo se distribuyen las valoraciones según autoubicación ideológica (escala de 1 a 10, izquierda-derecha). La calificación de "poca confianza" es común a todos los puntajes de autoubicación como

Tabla 3

Autoubicación (1-10: izquierda-derecha, gradualmente) $\times$ Confianza en la Administración de Justicia (\%)

\begin{tabular}{|l|c|c|c|c|c|c|c|c|c|c|c|c|c|}
\hline \multirow{2}{*}{ Confianza } & \multicolumn{10}{|c|}{ AUTOUBICACIÓN } & \multirow{2}{*}{ Total } \\
\cline { 2 - 13 } & $\mathbf{1}$ & $\mathbf{2}$ & $\mathbf{3}$ & $\mathbf{4}$ & $\mathbf{5}$ & $\mathbf{6}$ & $\mathbf{7}$ & $\mathbf{8}$ & $\mathbf{9}$ & $\mathbf{1 0}$ & $\mathbf{N . S .}$ & N.C. & \\
\hline Mucha & 3,0 & 2,1 & 3,5 & 4,5 & 5,9 & 2,4 & 3,5 & 12, & 5,6 &, 0 & 4,0 & 2,7 & 4,2 \\
\hline Bastante & 19,4 & 26,3 & 33,9 & 35,4 & 31,5 & 33,8 & 35,0 & 32,5 & 22,2 & 21,4 & 26,7 & 33,7 & 31,9 \\
\hline Poca & 50,7 & 50,5 & 50,1 & 47,0 & 45,4 & 47,6 & 46,2 & 35,0 & 33,3 & 42,9 & 39,4 & 40,1 & 44,9 \\
\hline Ninguna & 20,9 & 17,9 & 6,8 & 6,5 & 11,5 & 12,9 & 10,5 & 15,0 & 22,2 & 35,7 & 11,2 & 12,8 & 11,4 \\
\hline N.S. & 4,5 & 3,2 & 5,0 & 6,3 & 4,8 & 3,3 & 3,5 & 3,8 & 11,1 &, 0 & 18,4 & 8,6 & 6,8 \\
\hline N.C. & 1,5 &, 0 &, 6 &, 3 & 1,0 &, 0 & 1,4 & 1,3 & 5,6 &, 0 &, 4 & 2,1 &, 9 \\
\hline Total & 100,0 & 100,0 & 100,0 & 100,0 & 100,0 & 100,0 & 100,0 & 100,0 & 100,0 & 100,0 & 100,0 & 100,0 & 100,0 \\
\hline
\end{tabular}

de los medios de comunicación ha sido inmediatamente "mas-mediatizado". Tales circunstancias, sin embargo, deben incitarnos, más que descorazonarnos, hacia una apreciación crítica del Juicio. En tales áreas sensibles, los organismos internacionales deben proceder con el nivel más alto de exactitud en el desarrollo del razonamiento legal y en la determinación de los estándares requeridos de evidencia.)

${ }^{4}$ ESTUDIO CIS N. ${ }^{\circ} 2630$, BARÓMETRO DE DICIEMBRE de 2005 
la contestación más abundante, con una media muy alta: 44,9\%. En la tabla se han resaltado las puntuaciones por encima de las medias en cada valoración. Los porcentajes son interiores a cada casilla de autoubicación (porcentaje de gente con esa autoubicación que elige esa respuesta)

En la tabla 4 tenemos el cruce de "autoubicación" con la pregunta de percepción del trato (icree usted que en España a la hora de aplicar las leyes se da el mismo trato a todos, o se hacen diferencias según de quien se trate?) que nos indica con claridad una percepción generalizada del trato diferencial, que se hace más acusada a la izquierda de la escala. Los porcentajes son interiores a cada casilla de autoubicación (porcentaje de gente con esa autoubicación que elige esa respuesta).

En la tabla 5 tenemos el cruce de "autoubicación" con la pregunta de percepción del funcionamiento de los Tribunales de Justicia en España (Querría que me dijera ahora cómo considera usted que funcionan los Tribunales de Justicia en España: muy bien, bien, mal o muy mal). Los resultados generales son muy negativos, y se reparten de forma más o menos regular a ambos lados de la escala política, con alguna excepción.

Es decir, se trata de una percepción generalizada más allá de la autoubicación. Como lo es, en la tabla 6, la percepción de los medios con que cuentan los Tribunales de Justicia, y que parece que tiende a justificar o comprender su mal funcionamiento, al margen de los juicios a los jueces.

Pero en la tabla 7 vemos cómo son algunos más los que consideran que la independencia de los jueces es baja o muy baja frente a los que la consideran alta o muy alta, sin grandes diferencias a lo largo de la escala.

Y en la tabla 8 la situación se concreta (a través de frases que ayudan a matizar sobre situaciones habituales) de forma concluyente, tanto en lo que se refiere a la "objetividad" judicial como a la misma posibilidad de la Justicia, y es esto último lo que debería alarmar por encima de cualquier otra cosa.

Quizá el rango y la cifra más inquietante en torno a la Justicia vienen dados en la pregunta sobre malos tratos, en la tabla 9: ¿Cree Ud. que la mujer que denuncia malos tratos por parte de su pareja o ex pareja, puede tener mucha, bastante, poca o ninguna confianza en la ayuda que le puedan prestar los siguientes grupos o instituciones?:

La percepción popular es negativa en casi todo lo relativo a la Justicia, incluso bastante negativa. ¿Está inducida esta opinión por los medios o es resultado de una experiencia propia? Si tenemos en cuenta la Tabla 11, solo un $37,4 \%$ se han sentido incómodos o intimidados en los juzgados (vamos a tomarlo, a modo de supuesto razonable, como la concreción de un descontento más general). A su vez, solo un 35,9\% de encuestados han tenido algún contacto, directo o indirecto a través de amigos o familia, con la justicia (Tabla 10), con lo que se deduce que el número de descontentos reales (el 37,4\% del 35,9\%) es el 13,45\% de la población general mayor de edad, y esa es una cantidad inferior claramente a la de 
Tabla 4

Autoubicación (1-10: izquierda-derecha, gradualmente) $\times$ Percepción del trato

(\%)

\begin{tabular}{|l|c|c|c|c|c|c|c|c|c|c|c|c|c|}
\hline \multirow{2}{*}{ Trato } & \multicolumn{10}{|c|}{ AUTOUBICACIÓN } & \multirow{2}{*}{ Total } \\
\cline { 2 - 12 } & $\mathbf{1}$ & $\mathbf{2}$ & $\mathbf{3}$ & $\mathbf{4}$ & $\mathbf{5}$ & $\mathbf{6}$ & $\mathbf{7}$ & $\mathbf{8}$ & $\mathbf{9}$ & $\mathbf{1 0}$ & N.S. & N.C. & \\
\hline $\begin{array}{l}\text { Se da el } \\
\text { mismo } \\
\text { trato a } \\
\text { todos }\end{array}$ & 9,0 & 12,6 & 10,6 & 12,2 & 12,4 & 12,9 & 14,0 & 15,0 & 16,7 & 14,3 & 10,5 & 9,6 & 11,7 \\
\hline $\begin{array}{l}\text { Se hacen } \\
\text { diferen- } \\
\text { cias se- } \\
\text { gún de } \\
\text { quien se } \\
\text { trate }\end{array}$ & 91,0 & 86,3 & 86,1 & 83,6 & 84,4 & 82,9 & 81,1 & 82,5 & 83,3 & 85,7 & 82,3 & 84,2 & 84,1 \\
\hline N.S. &, 0 & 1,1 & 2,7 & 4,2 & 2,9 & 4,3 & 4,9 & 2,5 &, 0 &, 0 & 7,2 & 5,9 & 4,0 \\
\hline N.C. &, 0 &, 0 &, 6 &, 0 &, 4 &, 0 &, 0 &, 0 &, 0 &, 0 &, 0 &, 3 &, 2 \\
\hline Total & 100 & 100 & 100 & 100 & 100 & 100 & 100 & 100 & 100 & 100 & 100 & 100 & 100 \\
\hline
\end{tabular}

Tabla 5

Autoubicación (1-10: izquierda-derecha, gradualmente) $\times$ Percepción del funcionamiento de los Tribunales de Justicia en España

(\%)

\begin{tabular}{|l|c|c|c|c|c|c|c|c|c|c|c|c|c|}
\hline \multirow{2}{*}{ Funcionamiento } & \multicolumn{10}{|c|}{ AUTOUBICACIÓN } & \multirow{2}{*}{ Total } \\
\cline { 2 - 14 } & $\mathbf{1}$ & $\mathbf{2}$ & $\mathbf{3}$ & $\mathbf{4}$ & $\mathbf{5}$ & $\mathbf{6}$ & $\mathbf{7}$ & $\mathbf{8}$ & $\mathbf{9}$ & $\mathbf{1 0}$ & $\mathbf{N . S .}$ & N.C. & \\
\hline Muy bien & 1,5 &, 0 &, 0 &, 3 &, 4 &, 5 &, 7 &, 0 &, 0 &, 0 &, 7 &, 5 &, 4 \\
\hline Bien & 10,4 & 23,2 & 19,2 & 17,9 & 20,4 & 19,5 & 21,0 & 30,0 & 5,6 & 17,9 & 16,2 & 15,2 & 18,6 \\
\hline Regular & 25,4 & 33,7 & 35,4 & 39,6 & 36,3 & 34,8 & 39,9 & 27,5 & 22,2 & 21,4 & 33,6 & 37,2 & 35,6 \\
\hline Mal & 44,8 & 34,7 & 34,5 & 32,1 & 30,2 & 36,2 & 30,8 & 23,8 & 61,1 & 35,7 & 26,4 & 29,4 & 31,7 \\
\hline Muy mal & 10,4 & 3,2 & 5,0 & 4,5 & 6,3 & 5,2 & 6,3 & 11,3 & 11,1 & 17,9 & 6,5 & 5,9 & 6,1 \\
\hline N.S. & 6,0 & 5,3 & 5,6 & 5,7 & 6,1 & 3,3 & 1,4 & 7,5 &, 0 & 3,6 & 16,6 & 11,5 & 7,4 \\
\hline N.C. & 1,5 &, 0 &, 3 &, 0 &, 4 &, 5 &, 0 &, 0 &, 0 & 3,6 &, 0 &, 3 &, 3 \\
\hline Total & 100,0 & 100,0 & 100,0 & 100,0 & 100,0 & 100,0 & 100,0 & 100,0 & 100,0 & 100,0 & 100,0 & 100,0 & 100,0 \\
\hline
\end{tabular}


Tabla 6

Autoubicación (1-10: izquierda-derecha, gradualmente) $\times$ Percepción de los medios de los Tribunales de Justicia en España

(\%)

\begin{tabular}{|c|c|c|c|c|c|c|c|c|c|c|c|c|c|}
\hline \multirow{2}{*}{ Medios } & \multicolumn{12}{|c|}{ AUTOUBICACIÓN } & \multirow{2}{*}{ Total } \\
\hline & 1 & 2 & 3 & 4 & 5 & 6 & 7 & 8 & 9 & 10 & N.S. & N.C. & \\
\hline $\begin{array}{l}\text { Cuentan } \\
\text { con los } \\
\text { medios } \\
\text { necesarios }\end{array}$ & 23,9 & 22,1 & 27,4 & 22,6 & 26,5 & 22,4 & 25,9 & 18,8 & 33,3 & 28,6 & 25,3 & 22,7 & 24,6 \\
\hline $\begin{array}{l}\text { Les faltan } \\
\text { medios }\end{array}$ & 58,2 & 65,3 & 57,8 & 62,2 & 54,6 & 62,9 & 60,1 & 66,3 & 61,1 & 67,9 & 43,3 & 47,6 & 55,8 \\
\hline N.S. & 16,4 & 12,6 & 14,7 & 14,6 & 18,5 & 14,3 & 13,3 & 15,0 & 5,6 & 3,6 & 31,0 & 29,4 & 19,2 \\
\hline N.C. & 1,5 & ,0 & ,0 & ,6 & ,4 &, 5 &, 7 & ,0 & ,0 & 0 & ,4 & ,3 &, 4 \\
\hline Total & 100,0 & 100,0 & 100,0 & 100,0 & 100,0 & 100,0 & 100,0 & 100,0 & 100,0 & 100,0 & 100,0 & 100,0 & 100,0 \\
\hline
\end{tabular}

los que desconfían en general de la justicia y los jueces: cabe pensar que las diferencias son achacables a la transferencia desde los medios de una visión pesimista de la justicia. Creo que es así, aunque hay otras variables histórico-culturales que sin duda también están interviniendo en la percepción popular.

De alguna manera esa cantidad de descontentos reales que presuponemos nos sitúa en una estadística des-mediatizada, por volver a la expresión que usé al principio: una cantidad de ese orden debe ser la cifra de descontentos "puros".

La percepción excesivamente negativa de las instituciones de justicia no es directa, y debe provenir de variables culturales, históricas y mediáticas, sobre todo. Probablemente las mediáticas son las más fuertes, si tenemos en cuenta las cifras del seguimiento que cité al principio y que luego volveré a revisar. En este caso, y comenzando ya a hablar de los medios, cerraré este apartado con otra cita del importante caso ruandés desde el trabajo de Gabriele Della Morte, en la que la autora habla de una línea de demarcación que me parece de singular importancia: ...en otras palabras, debe existir una línea de la demarcación entre un análisis político que lleve a la defensa de una conciencia étnica y las expresiones que constituyen un crimen -un delito- ${ }^{5}$

\footnotetext{
${ }^{5}$ Della Morte, Gabriele (2005): p. 1129:

“One of the Media case's most innovative elements concerns the relationship between the crime of incitement (in a restricted sense) and the principle of freedom of expression (in a lateral sense). In other words, a demarcation line must be identified between a political analysis leading to an advocacy of an ethnic consciousness and speech which constitutes a crime." ${ }^{5}$ (subra-
} 
Tabla 7

Autoubicación (1-10: izquierda-derecha, gradualmente) $\times$ Percepción de la independencia de los jueces (grado)

(\%)

\begin{tabular}{|l|c|c|c|c|c|c|c|c|c|c|c|c|c|}
\hline \multirow{2}{*}{ Independencia } & \multicolumn{10}{|c|}{ AUTOUBICACIÓN } & \multirow{2}{*}{ Total } \\
\cline { 2 - 14 } & $\mathbf{1}$ & $\mathbf{2}$ & $\mathbf{3}$ & $\mathbf{4}$ & $\mathbf{5}$ & $\mathbf{6}$ & $\mathbf{7}$ & $\mathbf{8}$ & $\mathbf{9}$ & $\mathbf{1 0}$ & $\mathbf{N . S .}$ & $\mathbf{N . C .}$ & \\
\hline Muy alto & 4,5 & 4,2 & 4,7 & 1,5 & 4,8 & 2,4 & 3,5 & 6,3 &, 0 & 14,3 & 2,5 & 3,5 & 3,7 \\
\hline Bastante alto & 26,9 & 38,9 & 38,9 & 39,3 & 35,1 & 36,2 & 32,9 & 33,8 & 33,3 & 10,7 & 19,9 & 26,5 & 32,8 \\
\hline Bastante bajo & 32,8 & 33,7 & 33,9 & 35,4 & 34,4 & 40,0 & 37,1 & 30,0 & 38,9 & 42,9 & 28,9 & 27,3 & 33,3 \\
\hline Muy bajo & 16,4 & 12,6 & 5,9 & 3,9 & 6,9 & 5,2 & 7,0 & 7,5 & 16,7 & 10,7 & 4,3 & 5,3 & 6,3 \\
\hline N.S. & 13,4 & 9,5 & 15,6 & 19,3 & 17,4 & 14,3 & 19,6 & 17,5 & 5,6 & 14,3 & 44,0 & 34,2 & 22,2 \\
\hline N.S. & 6,0 & 1,1 &, 9 &, 6 & 1,5 & 1,9 &, 0 & 5,0 & 5,6 & 7,1 &, 4 & 3,2 & 1,7 \\
\hline Total & 100,0 & 100,0 & 100,0 & 100,0 & 100,0 & 100,0 & 100,0 & 100,0 & 100,0 & 100,0 & 100,0 & 100,0 & 100,0 \\
\hline
\end{tabular}

Tabla 8

Acuerdo o desacuerdo con diversas frases sobre la Administración de Justicia en España (\%)

\begin{tabular}{|l|c|c|c|c|}
\hline & $\begin{array}{c}\text { Las decisiones de } \\
\text { tribunales son } \\
\text { tan lentas, que } \\
\text { vale más evitar } \\
\text { meterse en } \\
\text { pleitos legales }\end{array}$ & $\begin{array}{c}\text { Los pleitos } \\
\text { legales son tan } \\
\text { caros que no } \\
\text { compensa acudir } \\
\text { a los tribunales }\end{array}$ & $\begin{array}{c}\text { En los pleitos, } \\
\text { 1os jueces suelen } \\
\text { actuar con } \\
\text { honradez, sin } \\
\text { dejarse influir } \\
\text { por ningún tipo } \\
\text { de presión }\end{array}$ & $\begin{array}{c}\text { Hoy día, el } \\
\text { resultado de un } \\
\text { pleito legal } \\
\text { depende, en } \\
\text { buena medida, } \\
\text { del juez que te } \\
\text { toque en cada } \\
\text { caso }\end{array}$ \\
\hline Muy de acuerdo & 29,7 & 21,6 & 4,5 & 19,8 \\
\hline De acuerdo & 50,3 & 47,9 & 27,3 & 50,4 \\
\hline $\begin{array}{l}\text { Ni de acuerdo ni en } \\
\text { desacuerdo }\end{array}$ & 5,3 & 8,8 & 19,3 & 9,8 \\
\hline En desacuerdo & 8,5 & 11,5 & 29,2 & 8,8 \\
\hline Muy en desacuerdo & 1,0 & 1,5 & 7,8 & 1,3 \\
\hline N.S. & 5,1 & 8,3 & 11,5 & 9,4 \\
\hline N.C. &, 2 &, 3 &, 4 &, 5 \\
\hline
\end{tabular}


Tabla 9

¿Cree Ud. que la mujer que denuncia malos tratos por parte de su pareja o ex pareja, puede tener mucha, bastante, poca o ninguna confianza en la ayuda que le puedan prestar los siguientes grupos o instituciones?:

(\%)

\begin{tabular}{|l|c|c|c|c|c|c|c|c|c|c|}
\hline & $\begin{array}{c}\text { El } \\
\text { Estado }\end{array}$ & $\begin{array}{c}\text { La } \\
\text { policía }\end{array}$ & $\begin{array}{c}\text { Los } \\
\text { aboga- } \\
\text { dos }\end{array}$ & $\begin{array}{c}\text { Los } \\
\text { servicios } \\
\text { médicos }\end{array}$ & $\begin{array}{c}\text { Los } \\
\text { servicios } \\
\text { sociales }\end{array}$ & $\begin{array}{c}\text { Los } \\
\text { juzgados } \\
\text { jueces) }\end{array}$ & $\begin{array}{c}\text { Los } \\
\text { medios } \\
\text { de co- } \\
\text { munica- } \\
\text { ción }\end{array}$ & $\begin{array}{c}\text { La } \\
\text { famila }\end{array}$ & $\begin{array}{c}\text { Las or- } \\
\text { ganiza- } \\
\text { amigos/as } \\
\text { ciones } \\
\text { de mu- } \\
\text { jeres }\end{array}$ \\
\hline Mucha & 6,5 & 10,0 & 7,5 & 21,7 & 20,9 & 5,6 & 12,1 & 35,7 & 27,2 & 36,0 \\
\hline Bastante & 33,8 & 44,4 & 43,8 & 60,8 & 56,9 & 34,0 & 43,2 & 47,0 & 50,7 & 49,7 \\
\hline Poca & 41,8 & 32,5 & 31,0 & 10,0 & 13,2 & 41,3 & 26,1 & 8,0 & 11,8 & 5,3 \\
\hline Ninguna & 9,4 & 6,6 & 7,6 & 1,5 & 1,8 & 10,8 & 9,6 & 1,1 & 1,6 &, 9 \\
\hline N.S. & 7,6 & 5,7 & 9,0 & 5,5 & 6,4 & 7,1 & 7,8 & 6,5 & 7,3 & 7,5 \\
\hline N.C. &, 8 &, 8 & 1,2 &, 6 &, 8 & 1,3 & 1,2 & 1,6 & 1,5 &, 7 \\
\hline
\end{tabular}

Tabla 10

¿Ha tenido alguna vez contacto con el mundo de la Justicia (como demandante, demandado, testigo, miembro de un jurado, etc.)? Nos referimos a contacto directo o como acompañante de alguien de su familia o amigos.

(\%)

\begin{tabular}{|l|c|}
\hline Sí & 35,9 \\
\hline No & 64,0 \\
\hline N.C. &, 1 \\
\hline Total & 100,0 \\
\hline
\end{tabular}

Quizá esa línea de demarcación sea, finalmente, objeto de debate entre el sistema judicial, los políticos y los periodistas, pero es evidente que en nuestro país esa línea es irregular y desconoce con frecuencia las diferencias entre la libertad de expresión y la incitación a la desconfianza de la justicia más allá de lo razonable y de forma o modo poco razonable. Esa es la cuestión última, y como siempre se trata de un tema de consenso nunca bien cerrado.

yado mío): (Uno de los elementos más interesantes del caso de los medios concierne a las relaciones entre la incitación al crimen -el delito de incitación al crimen- (en un sentido restringido) y el principio de libertad de expresión (en un sentido lateral). En otras palabras, debe existir una línea de la demarcación entre un análisis político que lleve a la defensa de una conciencia étnica y las expresiones que constituyen un crimen -un delito-.) 
Tabla 11

(Sólo a quienes han tenido contacto personal con el mundo de la justicia) $Y$ cuando fue a los juzgados, se sintió Ud...

\begin{tabular}{|l|c|}
\hline & $\mathbf{( \% )}$ \\
\hline Cómodo, bien atendido & 23,2 \\
\hline $\begin{array}{l}\text { Normal, como en cualquier otra } \\
\text { oficina pública }\end{array}$ & 37,9 \\
\hline Incómodo, intimidado & 37,4 \\
\hline N.S. &, 1 \\
\hline N.C. & $\mathbf{1 , 3}$ \\
\hline Total & 100 \\
\hline
\end{tabular}

\section{La justicia en la agenda pública}

Pero hay un dato en el Barómetro del CIS que estamos analizando que parece contradictorio con todo esto: "La Administración de Justicia" apenas es un problema para los encuestados $(2,4 \%$ de respuestas) en la llamada "pregunta de agenda pública” (¿Cuáles son, a su juicio, los tres problemas principales que existen actualmente en España? -máximo tres respuestas-), ocupando el puesto 16 en el conjunto de las respuestas-rango. Nunca ocupó un papel relevante, y pocas veces figuró entre los problemas de los 15 primeros rangos en los últimos años (ver gráficos 3 y 4).

Esto podría querer decir que las presiones y la insistencia mediática sobre la Justicia no se transfieren a la Agenda Pública, y por lo tanto no constituyen un problema de alcance electoral, por ejemplo. Los temas relevantes de la Agenda Pública (Paro, Terrorismo, Inmigración, Vivienda, Inseguridad Ciudadana, Economía... etc) se constituyen en el eje de referencia para la acción política.

Cabría pensar que la Justicia se solapa en los otros temas (terrorismo sobre todo), y así aparece en nuestro estudio, en el que la mayoría de los que he llamado vínculos judiciales lo son sobre temas terroristas. Por tanto, la gente "concreta" los problemas de la justicia en su variedad temática, y esto le resta fuerza al tema "justicia” en sí mismo.

Al no producirse una situación que coloque al ítem o issue o tema Justicia como un problema relevante entre los máximos rangos (le doy esta consideración, por su relevancia estadística, a los tres primeros rangos, sobre todo) de la Agenda pública (ni de la Agenda personal, que es aquella en que se sustituyen los problemas del país por los personales en la pregunta de agenda), no se puede hablar en ningún caso de área de impacto (relaciones entre los tres primeros rangos de ambas agendas y que podrían marcar la importancia de ciertos temas en ciertas situaciones para ser especialmente percibidos 


\section{Gráfico 3}

\% de elección del problema JUSTICIA de 2000-2006

Datos $\mathrm{CE}$

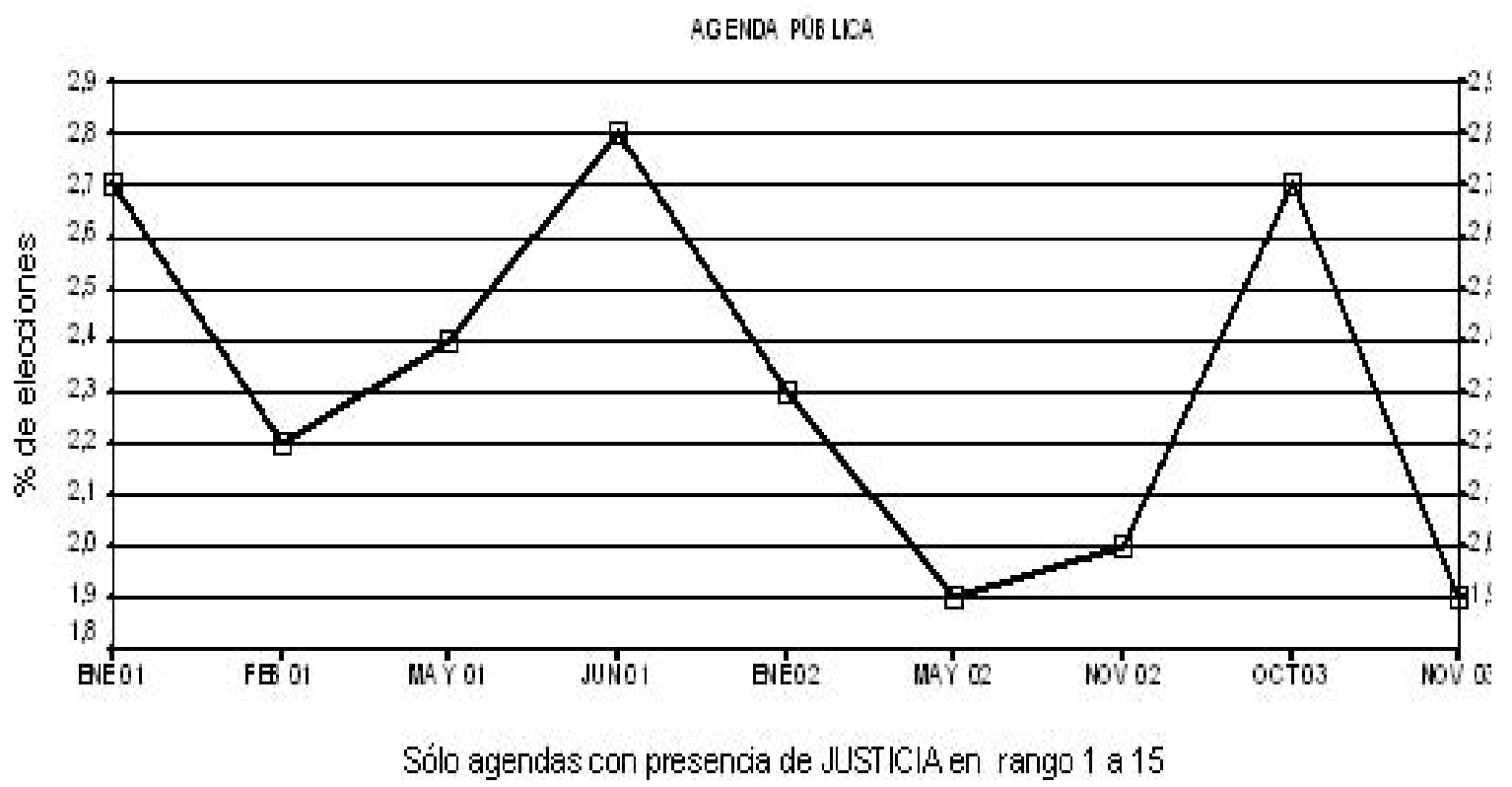

por la opinión pública en el contexto de los procesos de comunicación pública y política), concepto que desarrollo en otro trabajo y que espero poder desarrollar en otros artículos sobre nuevas posibilidades analíticas de la teoría de la Agenda Setting.

Con todo, el dato, que es contradictorio con los pronunciamientos generales sobre la Administración de Justicia, no lo es con el dato de "solo" un 13,45\% de descontentos reales con tal administración. Todo parece conducir, al menos, a una cuádruple conclusión:

1. Los descontentos concretos con la justicia son alrededor de un $13,45 \%$ de la población general (tomando como referencia las preguntas citadas y los supuestos que contienen), y el resto de descontentos lo son desde consideraciones más abstractas que no los lleva a considerar a la Justicia un problema en la pregunta de Agenda Pública ${ }^{6}$.

\footnotetext{
${ }^{6}$ La teoría de la agenda-setting ha tratado con particular rigor las transferencias entre la agenda mediática y la agenda pública.
} 


\section{Rango de elección del problema JUSTICIA de 2000-20006}

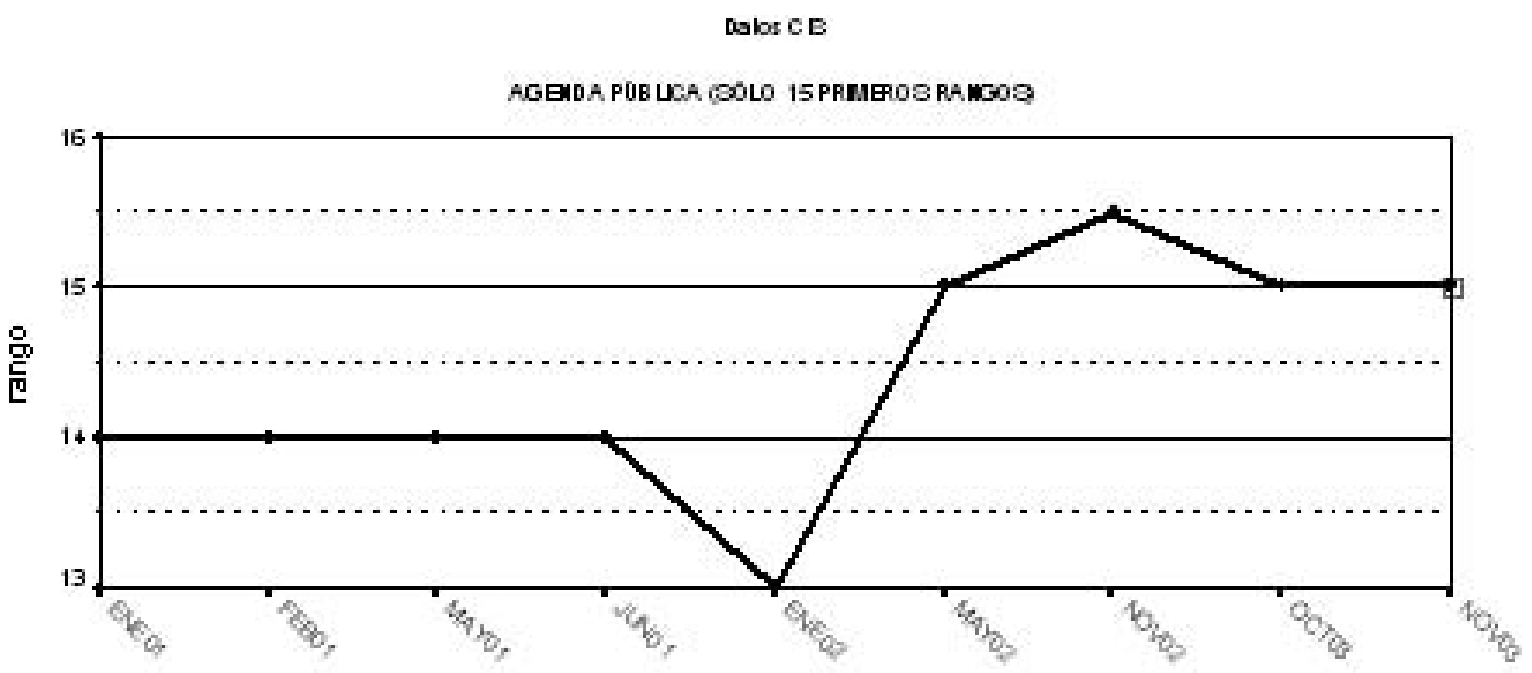

Fecha

2. Es posible que una parte sustancial del descontento con la Justicia se solape con otros temas en la Agenda Pública, en particular con el terrorismo.

3. Con todo, parece haber un exceso de descontentos que nunca han tenido contacto con la justicia concreta, lo que hace pensar en una fuerte influencia mediática que "enmarca" (framing) muchas opiniones populares sobre la justicia, en particular las de aquellos que nunca han tenido que recurrir a ella.

4. Hay estilos/ritmos de información sobre la justicia, auténticos Bloques de Opinión Público-Mediática (que se convertirán, según y cómo, en opinión pública general), que, en el caso de este estudio, se muestran en forma de importantes correlaciones entre algunos medios (El País/El Periódico) en las series numéricas de informaciones judiciales en portada/fecha (ver tabla 12).

\section{Los efectos de los medios y la fortaleza del método}

Por las razones expresadas a partir de los datos manejados, todo hace pensar en una influencia importante de los medios sobre la opinión pública y, quizá, sobre los mismos jueces y el resto de la admi-

El trabajo original es el de: McCombs y Shaw (1972). Existen numerosos textos sobre efectos de los medios, el de Roda Fernandez, R. (1989). sería uno de especial interés y rigor. 
Tabla 12

Correlaciones de las series de cada diario

del $\mathbf{n}^{\circ}$ de noticias judiciales/fecha

\begin{tabular}{|l|l|c|c|c|c|}
\hline \multirow{4}{*}{ EL PAÍ́S } & & EL PAÍS & ABC & EL PERIÓDICO & EL MUNDO \\
\hline \multirow{4}{*}{ ABC } & C. Pearson & 1 &, 449 &, $644\left(^{* *}\right)$ &, 129 \\
\cline { 2 - 6 } & Sig. (bilateral) & &, 093 &, 010 &, 646 \\
\cline { 2 - 6 } & $\mathrm{N}$ & 15 & 15 & 15 & 15 \\
\hline \multirow{3}{*}{ EL PERIÓDICO } & C. Pearson &, 449 & 1 &, 075 &, 352 \\
\cline { 2 - 6 } & Sig. (bilateral) &, 093 &, 790 &, 199 & 15 \\
\cline { 2 - 6 } & $\mathrm{N}$ & 15 & 15 & 15 &, 076 \\
\hline \multirow{3}{*}{ EL MUNDO } & C. Pearson &, $644\left(^{* *}\right)$ &, 075 & 1 &, 788 \\
\cline { 2 - 6 } & Sig. (bilateral) &, 010 &, 790 & & 15 \\
\cline { 2 - 6 } & $\mathrm{N}$ & 15 & 15 & 15 & 1 \\
\cline { 2 - 6 } & C. Pearson &, 129 &, 352 &, 076 & 15 \\
\cline { 2 - 6 } & Sig. (bilateral) &, 646 &, 199 & 15 & 158 \\
\cline { 2 - 6 } & $\mathrm{N}$ & 15 & 15 & 15 & \\
\hline
\end{tabular}

** La correlación es significativa al nivel 0,01 (bilateral).

nistración de Justicia. Esto forma parte de los regímenes democráticos de opinión, y es normal y necesario, aunque es preciso que la gente y los administradores de la justicia lo tengan en cuenta tanto a la hora de opinar sobre la justicia como a la hora de ejecutarla: no hay nada fuera del complejo sistema de interacciones que forma la vida social, la sociedad misma, y si la objetividad científica es, sobre todo, una cuestión de método, la objetividad y la independencia judiciales deberían arraigarse, sobre todo, en la solidez metodológica de todo proceso para fundar cualquier decisión sobre cuestiones corroborables y, por tanto, debatibles y recurribles, más allá de la inevitable ecuación personal que los astrofísicos llegan, incluso, a cuantificar, y que da cuenta de lo más subjetivo de nuestras decisiones, que en todo caso, si son metodológicamente correctas, deben ser, insisto, corroborables y debatibles. O, en lenguaje jurídico, recurribles, con todo lo que eso acarrea de posibilidad de revisión objetiva (metodológicamente adecuada) de los procesos de construcción de la sentencia. O, como hoy se dice: toda actividad judicial debe ser factible de de-construcción, y eso es la fortaleza del método. 


\section{Referencias bibliográficas}

- Della Morte, G. (2005): "De-Mediatizing the Media Case", Journal of International Criminal Justice, vol. 3, n. ${ }^{\circ} 4$, pp. 1019-1033.

- McCombs, M; Shaw, D. (1972): “The Agenda-Setting Function of Mass Media”. Public Opinion Quarterly, vol. 36, n. ${ }^{\circ}$, pp. 176-187. (ver también, en castellano y en edición on-line)

- Roda Fernandez, R. (1989): Medios de comunicación de masas. Su influencia en la sociedad y en la cultura contemporáneas, Madrid: Siglo XXI/CIS. 